\title{
Absolute judgment of more than 32 categories (5 bits) with a unidimensional visual stimulus'
}

HERBERT FRIEDMAN

COLLEGE OF WILLIAM AND MARY

In a series of four experiments using line length and bisected length stimuli, performance was well above the commonly accepted maximum of nine to 15 categories. Channel capacity (measured by an information analysis) for the median Ss in the last two studies was 28 and 45 categories. These results suggest that previous research on channel capacity should be re-examined.

Miller (1956), reviewing research on information processing and perception, pointed out that the number of stimuli a person can correctly identify on a unidimensional stimulus continuum appears to have a maximum in the range of nine to 15 . Subsequent studies of a variety of stimulus dimensions have consistently confirmed this conclusion (McCormick, 1964; Gibson, 1963). This rather limited capacity for the absolute identification of stimuli has raised numerous theoretical and practical problems (Liberman, 1957; Miller, 1956). The following report describes four studies in which unaided performance with two different types of line length stimuli was markedly superior to that previously reported.

Table 1 presents the details of the number of observers (Os), stimulus type and range, number of training sessions, and the performance of the median $O$ on the final training day for each experiment. The data was subjected to an information analysis (Garner \& Hake, 1951) and the results for information transmitted are expressed in bits and categories. This is essentially the same analysis as used in the studies referred to by Miller (1956) and McCormick (1964). All Os were undergraduate students at the College of William and Mary who volunteered and were paid for participation. No student participated in more than one experiment. Each daily session required approximately 15 to 20 min for Experiments 1 through 3, and $30 \mathrm{~min}$ for Experiment 4 .

In Experiment 1 the apparatus was a featureless black panel, $100 \mathrm{~cm}$ long and $64 \mathrm{~cm}$ high in which a horizontal slot $(60 \mathrm{~cm} \times 5 \mathrm{~cm})$ permitted exposure of a black wooden dowel (1 cm diameter) set on a white background. Horizontal sliding doors between the rod and front panel enabled the $E$ to manipulate the exposed length of the rod. The 30 stimulus lengths varied from $1.0 \mathrm{~cm}$ to $26.85 \mathrm{~cm}$ with a constant ratio of 1.2 between successive stimuli. Five different settings of the left hand slide (i.e., five positions for the zero point) prevented successful use of panel cues in judgment. The longest rod length shown was less than half the size of the slot to require the Os to judge with reference to the left hand or zero position. A vertical sliding door placed in front of the slot masked the setting of the horizontal doors between trials. Normal room lighting was used and the Os were seated approximately $1 / 2 \mathrm{~m}$ from the panel. The 30 stimulus positions were labeled in order from 1 to 30 , and $O$ was instructed to give the number of each stimulus as it was presented. The correct answer would then be announced and $O$ was to repeat this. Each line length was then presented three times in random order. The five zero positions were also varied randomly from trial to trial. The same procedure was used for all subsequent days with all stimuli being presented and labeled by $E$ at the start of each session. Performance on the last day for the median $O$ was equivalent to 19.2 absolute identifications, and was markedly superior to that originally anticipated.

We therefore decided to investigate another type of line length discrimination, one using a bounded stimulus length (i.e., one in which both end points are presented and the stimulus is the length marked off by an intervening point). The unbounded line may be a difficult stimulus continuum since there are no clearcut reference points for any of the stimuli. In contrast, the bounded stimulus length provides both a precise reference length given by the end points and the opportunity for the $O$ to identify stimuli by bisecting or trisecting the range.

Experiment 2 used the same apparatus as in Experiment 1 except that the wooden dowel and the white background were removed from the panel and three $1 / 10-W$ NE-2 neon bulbs were placed within the open slot. The two end bulbs were stationary during the experiment while the middle bulb was

Table 1. Experimental conditions and the final day performance for the median 0 for each group. Experiment 1 employed an unbounded line stimulus and Experiments 2, 3, and 4 employed a bounded length stimulus.

\begin{tabular}{rrrrrrrr} 
Exp. N & $\begin{array}{c}\text { Stim. } \\
\text { range } \\
\text { in cm. }\end{array}$ & $\begin{array}{c}\text { Stim. } \\
\text { cat. }\end{array}$ & $\begin{array}{c}\text { No. of } \\
\text { training } \\
\text { sessions }\end{array}$ & $\begin{array}{r}\text { Final day performance } \\
\text { of median } 0\end{array}$ \\
\hline 1 & 9 & 26.85 & 30 & 20 & 4.26 & 19.2 & 68.3 \\
$2 \mathrm{E}$ & 11 & 26.50 & 20 & 10 & 4.12 & 17.4 & 96.7 \\
$2 \mathrm{C}$ & 7 & 26.50 & 20 & 10 & 4.06 & 16.6 & 91.6 \\
3 & 7 & 25.00 & 50 & 10 & 4.81 & 28.1 & 59.6 \\
4 & 5 & 50.00 & 100 & 15 & 5.48 & 44.6 & 34.8 \\
\hline
\end{tabular}


movable. The left hand light was labeled the zero point and the right hand light was labeled 100. The stimulus was the distance between the left hand light and the movable marker light as a percentage of the total length. Thus, the marker light positioned directly between the two end bulbs represents the stimulus 50. During exposure of the stimulus there was no other light in the room. Normal room illumination between trials prevented dark adaptation. The stimulus panel was kept at a constant distance $(1.33 \mathrm{~m})$ from $O$. It was necessary to increase the observing distance from that used in Experiment 1 in order that the neon bulbs would appear as virtual point sources. After introducing the Os to their task and showing the 19 stimuli (neither the zero nor 100 stimulus was used), two sets of instructions were employed. Os in the experimental (E) group were given the correct number of the stimulus after each response and had to repeat the correct response. Os in the control group (C) were given no information concerning the accuracy of their responses. Each daily session consisted of the presentation of the 19 lengths three times each in a semi-random order. At the start of each daily session the Os were shown the 19 stimuli and each was labeled by $E_{\text {. Median }}$ performance at the level of 15 to 17 eategories for both groups was comparable to that observed in the experiment with the unbounded line stimulus. Group C showed appreciable improvement during training which suggests that they are capable of learning to divide the total length into equal segments. However, the experimental group was consistently superior to the control group, indicating that knowledge of the correct response does aid in this task (probably by minimizing constant errors). Two Os in Group $\mathrm{E}$ and one in Group $C$ made no errors on the last day and the task was clearly not a difficult one.

In Experiment 349 stimuli ( 2 to 98 ) were used with the same procedure as in Experiment 2, Group E. The performance of the median $O$ on the 10th day was considerably better than that obtained in Experiment 2. The level of almost five bits for the information measure agrees with the percent correct (i.e., $60 \%$ of 50 categories) and is larger than that reported for some multidimensional stimulus displays (e.g., Eriksen \& Hake, 1955). Information processing at this rate is analogous to efficiently reading a dial or speedometer without using the numerical scale markings.

Experiment 4 employed the same size stimuli with twice the number of categories (100) and a doubled stimulus range $(50 \mathrm{~cm})$. The procedure was the same as that in Experiment 3 except that 15 days of training were 'ised, since on this task the Os were still improving at Day 10. On the last day the median $O$ was performing at a level equivalent to 45 categories. Earlier studies with length and size stimuli (Eriksen \& Hake, 1955) found no increase in information processed with increased stimulus range or increased number of categories. Miller (1955) pointed out that information measures tend to overestimate as the number of stimuli increases. Such a bias would account for the disparity between the number of categories and percentage correct in Experiment 4.

These findings raise the immediate question of possible reasons for the marked differences between the performance observed here and in previously reported studies. Comparable results in the two different types of length tasks indicate that possible unique aspects of either situation are not suitable explanations for the present findings. After the last session all os were briefly interviewed to determine if any extraneous cues were used in making the judgments; no artifacts or additional cues were revealed. The bounded length task can readily be seen to be a very simple one. A naive $O$ can usually make $75-90 \%$ correct judgments in a bounded length task when using 20 intervals between zero and 100 and make no errors with 10 intervals. Vertical lines or dots drawn on paper would serve as adequate stimuli for a simple demonstration of this phenomenon. The lack of equivalent findings in preceding studies is not readily explained. In similar experiments inadequate stimulus displays or insufficient training may have prevented the Os from performing at their maximum level.

These findings suggest the need to re-examine the generality of Miller's conclusion concerning the maximum capacity to process information in an absolute identification task. Indeed, it appears that people may do surprisingly well rather than surprisingly poorly in this type of problem.

\section{References}

Eriksen, C. W., \& Hake, H. W. Multidimensional stimulus differences and accuracy of discrimination. J. exp. Psychol., 1955, $50,153-160$.

Eriksen, C. W., \& Hake, H. W. Anchor effects in absolute judgments. J. exp. Psychol., 1957, 53, 132-138.

Garner, W. R., \& Hake, H. W. The amount of information in absolute judgments. Psychol. Rev., 1951, 58, 446-459.

Gibson, Eleanor J. Perceptual learning. Annu. Rev. Psychol., 1963, $14,29-58$.

Hake, H. W., \& Gamer, W. R. The effect of presenting various numbers of discrete steps on scale reading accuracy. $J$. exp. Psychol., 1951, 42, 358-366.

Klemmer, E. T., \& Frick, F. C. Assimilation of information from dot and matrix patterns. J. exp. Psychol., 1953, 45, 15-19.

Liberman, A. N. Some results of research on speech perception. J. Acoust. Soc. Amer., 1957, 29, 117-123.

McCormick, E. J. Human factors engineering. New York: McGraw Hill, 1964. Pp. 83-124.

Miller, G. A. The magical number seven, plus or minus two: some limits on our capacity for processing information. Psychol. Rev., $1956,63,89-97$.

\section{Note}

1. Supported in part by NIH Grant MH 10532-01, NASA Contract NAS 1-4738 and NASA Grant NGR 47-006-028. Portions of these data were presented at the Virginia Academy of Science meeting May, 1966 (Va. J. Sci., 1966, 17, 360). I wish to thank Franklin McLean, William Ewald, Robert Zemore, and Patricia Hubbell for their assistance in collecting and analyzing the data. 